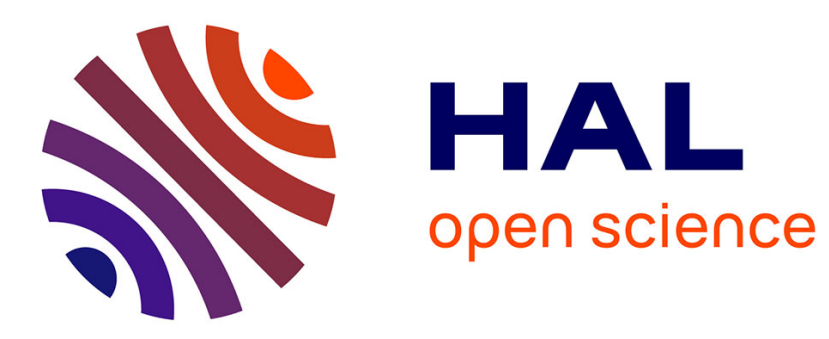

\title{
Modified Kolsky Formulas for an Increased Measurement Duration of SHPB Systems
}

Gérard Gary, Dirk Mohr

\section{To cite this version:}

Gérard Gary, Dirk Mohr. Modified Kolsky Formulas for an Increased Measurement Duration of SHPB Systems. Experimental Mechanics, 2013, 53 (4), pp.713-717. hal-00968942

\section{HAL Id: hal-00968942 \\ https://hal.science/hal-00968942}

Submitted on 4 Apr 2014

HAL is a multi-disciplinary open access archive for the deposit and dissemination of scientific research documents, whether they are published or not. The documents may come from teaching and research institutions in France or abroad, or from public or private research centers.
L'archive ouverte pluridisciplinaire HAL, est destinée au dépôt et à la diffusion de documents scientifiques de niveau recherche, publiés ou non, émanant des établissements d'enseignement et de recherche français ou étrangers, des laboratoires publics ou privés. 


\title{
Modified Kolsky Formulas for an Increased Measurement Duration of SHPB Systems
}

\author{
Gérard Gary ${ }^{1}$ and Dirk Mohr ${ }^{1,2}$ \\ ${ }^{1}$ Solid Mechanics Laboratory (CNRS-UMR 7649), Department of Mechanics, \\ École Polytechnique, Palaiseau, France \\ ${ }^{2}$ Impact and Crashworthiness Laboratory, Department of Mechanical Engineering, \\ Massachusetts Institute of Technology, Cambridge MA, USA
}

\begin{abstract}
The so-called incident, reflected and transmitted strain histories are typically recorded during standard Split Hopkinson Pressure Bar (SHPB) experiments. Subsequently, the stress-strain curve for the specimen material is determined based on these recordings. Unless wave deconvolution techniques are employed, the reliable measurement of the reflected wave requires an input bar which is at least twice as long as the striker bar (of equal impedance). The present brief technical note elucidates the advantages of a simple alternative configuration which has only been seldom used in the past. Based on the assumption of quasi-static equilibrium at the specimen level, we present a modification of Kolsky's formulas such that the stress-strain curve for the specimen material can be obtained from the measurement of the incident and transmitted strain histories only. As a result, the measurement of the reflected wave may be omitted and a much shorter input bar can be chosen. Conversely, a much longer striker bar may be used for a given input bar length, thereby increasing the valid duration of standard SHPB experiments by up to $100 \%$ through the use of the modified Kolky formulas. An example experiment is shown where the duration of valid measurements has been increased by more than $70 \%$.
\end{abstract}

Keywords: Split Hopkinson pressure bar, Kolsky formulas, high strain rate testing 


\section{Motivation}

Split Hopkinson Pressure Bar (SHPB) systems are used to investigate the mechanical behavior of materials at high strain rates. The widespread use of SHPB systems in experimental dynamics is mainly due to the simplicity of the experimental procedure. The experimental technique is based on the early work of Hopkinson (1913), who recorded a pressure-pulse profile using a slender bar. This approach has been widely adopted since the in-depth analysis of Davies (1948). The practical configuration consisting of a short specimen sandwiched between two slender bars is due to Kolsky (1949). High impedance bars made of steel are typically employed to perform dynamic experiments on metals. After being initially developed for compression tests, the technique was soon extended to tensile loading by Harding et al. (1960 and to torsion loading by Duffy et al. (1971). A comprehensive review of developments in classical SHPB testing has been provided by Gray III (2000).

The stress-strain relationship for the specimen material in a SHPB experiment is typically determined based on the so-called incident, reflected and transmitted waves. It is common practice to measure the incident and reflected waves using a strain gage at the center of the input bar. This imposes a constraint on the minimum length of the input bar to avoid the superposition of the incident and reflected waves at the position of the strain gage. For example, when both bars have the same diameter and are made from the same material, the input bar needs to be at least twice as long as the striker bar to guarantee the validity of the input bar strain history measurements. This limitation can be overcome through the use of multi-point measurements along with a wave deconvolution technique (e.g. Bussac et al. (2002)). A valuable alternative approach omitting the use of wave deconvolution has been used by Song et al. (2007); they positioned the input bar strain gage near the impact end of the input bar to double the duration of the valid loading pulse duration. It is the purpose of our technical note to elucidate the advantages of this simple alternative configuration. It is presented as a modification of Kolsky's formulas for the use of much shorter input bars without sacrificing measurement accuracy or changing the length and magnitude of the loading pulse. 


\section{Modified Kolsky formulas}

Figure 1 shows the set-up of a SHPB system composed of an elastic striker bar, input bar, specimen and output bar. We differentiate among the corresponding bar lengths $l_{i}$, cross-sections $A_{i}$, Young's moduli $E_{i}$ and wave propagation velocities $c_{i}$ through the subscript $i$, with $i=s t$, in, out for the striker, input and output bar, respectively. As the striker bar hits the input bar, the rightward travelling incident strain wave $\varepsilon_{i n c}(x, t)$ is created in the input bar. It is partially reflected and transmitted at the input bar specimen interface. As a result, the leftward travelling reflected wave $\varepsilon_{r e}(x, t)$ is observed in the input bar, while a rightward travelling transmitted wave $\varepsilon_{t r a}(x, t)$ is created in the output bar. Based on the strain histories $\bar{\varepsilon}_{\text {inc }}(t)=\varepsilon_{\text {inc }}\left(x_{i n}, t\right)$ and $\bar{\varepsilon}_{r e}(t)=\varepsilon_{r e}\left(x_{i n}, t\right)$ at the input bar-specimen boundary $\left(x=x_{i n}\right)$, we may compute the input velocity and force,

$$
\begin{aligned}
& v_{i n}=-c_{i n}\left(\bar{\varepsilon}_{i n c}-\bar{\varepsilon}_{r e}\right) \\
& F_{i n}=E_{i n} A_{i n}\left(\bar{\varepsilon}_{i n c}+\bar{\varepsilon}_{r e}\right) .
\end{aligned}
$$

Analogously, we may compute the output velocity and force based on the strain history $\bar{\varepsilon}_{\text {out }}(t)=\varepsilon_{\text {tra }}\left(x_{\text {out }}, t\right)$ at the output bar-specimen boundary $\left(x=x_{\text {out }}\right)$,

$$
\begin{aligned}
& v_{\text {out }}=-c_{\text {out }} \bar{\varepsilon}_{\text {tra }} \\
& F_{\text {out }}=E_{\text {out }} A_{\text {out }} \bar{\varepsilon}_{\text {tra }} .
\end{aligned}
$$

These three strain histories must be clearly identified based on the experimental measurement. The striker impedance must be smaller or equal to that of the input bar (striker rebound) and its length must be less than $50 \%$ of that of the input bar.

In a standard SHPB experiment, the assumption of quasi-static specimen equilibrium

$$
F_{\text {in }}(t) \cong F_{\text {out }}(t)
$$


needs to hold true. Assuming a homogeneous distribution of the axial stress and strain fields within the specimen, the engineering stress and strain rate histories within the specimen are approximated by the so-called three-wave formulas

$$
\begin{aligned}
& \sigma(t)=\frac{E_{\text {out }} A_{\text {out }} \bar{\varepsilon}_{\text {tra }}}{A_{s}} \\
& \dot{\varepsilon}(t)=\frac{c_{\text {in }}\left(\bar{\varepsilon}_{\text {inc }}-\bar{\varepsilon}_{r e}\right)-c_{\text {out }} \bar{\varepsilon}_{\text {tra }}}{l_{s}},
\end{aligned}
$$

with $A_{s}$ and $l_{s}$ denoting the specimen cross-sectional area and length, respectively. From a signal processing point of view, the assumption of quasi-static equilibrium (5) may be exploited to obtain a relationship among the strain histories at the specimen boundaries. For a standard system with three bars of identical diameters and material properties, we have

$$
\bar{\varepsilon}_{i n c}(t)+\bar{\varepsilon}_{r e}(t) \cong \bar{\varepsilon}_{t r a}(t),
$$

while for the more general case of bars of different impedance, the above equation reads

$$
\bar{\varepsilon}_{i n c}(t)+\bar{\varepsilon}_{r e}(t) \cong \lambda \bar{\varepsilon}_{\text {tra }}(t) \quad \text { with } \quad \lambda=\frac{E_{\text {out }} A_{\text {out }}}{E_{\text {in }} A_{\text {in }}}
$$

Kolsky (1949) made use of Eq. (8a) to express the incident wave as a function of the reflected and transmitted strain histories, leading to the "standard Kolsky formulas" which express the stress and strain in the specimen as a function of the reflected and transmitted wave only. As an alternative to removing the incident wave from the final formulas, we make use of Eq. (8) to come up with modified Kolsky formulas for the stress and strain history in the specimen that do not require knowledge of the reflected wave. For standard case of identical bars, we have

$$
\begin{gathered}
\sigma(t)=\frac{E A \bar{\varepsilon}_{t r a}}{A_{s}} \\
\dot{\varepsilon}(t)=\frac{c\left(\bar{\varepsilon}_{i n c}-\bar{\varepsilon}_{t r a}\right)}{l_{s}},
\end{gathered}
$$

while for the general case, the modified Kolsky formulas read 


$$
\begin{gathered}
\sigma(t)=\frac{E_{o u t} A_{o u t} \bar{\varepsilon}_{\text {tra }}}{A} \\
\dot{\varepsilon}(t)=\frac{2 c_{\text {in }} \bar{\varepsilon}_{\text {inc }}-\left(\lambda c_{\text {in }}+c_{\text {out }}\right) \bar{\varepsilon}_{\text {tra }}}{l}
\end{gathered}
$$

This modification is of great advantage from an experimental point of view. In the case of the three-wave and standard Kolsky's formulas, the reflected wave needs to be measured which imposes a much stronger constraint on the validity of the strain history measurement as compared to the new approach, where the measurement of the incident wave is sufficient (in addition to the transmitted wave). Reliable measurements of the incident wave may be obtained from a strain gage positioned near the striker-input bar interface for a duration of approximately $\Delta t=2 l_{i n} / c_{i n}$. Furthermore, striker rebound is no longer required and its impedance may even be greater than that of the input bar. The duration of valid stress-strain curve measurements is therefore greatly increased (by a factor of two in theory) for a given input bar length when using the modified Kolsky formulas. It is emphasized that the above formulas are only valid if the assumption of quasi-static equilibrium holds true. For example, the assumption of quasi-static equilibrium is often not satisfied at the beginning of SHPB experiments or when testing brittle or low impedance materials.

\section{Design of compact SHPB systems}

Based on the modified Kolsky formulas, a compact SHPB system can be designed as follows:

1. Chose the duration $T$ of the experiment. It is usually defined through the desired maximum strain $\varepsilon_{\max }$ and the average strain rate $\dot{\varepsilon}, T=\varepsilon_{\max } / \dot{\varepsilon}$.

2. Determine the corresponding striker bar length $l_{s t}=c_{s t} T / 2$;

3. Determine the input bar length $l_{i n}=5 D_{i n}+c_{i n} T / 2$, assuming that the input bar strain gage is positioned at a distance of $5 D_{\text {in }}$ from the striker-input bar 
interface, with $D_{\text {in }}$ denoting the input bar diameter (see for instance [9] for a recommendation of the required minimum distance).

4. Determine the output bar length $l_{\text {out }}=5 D_{\text {out }}+c_{\text {out }} T / 2$.

As illustrated in Fig.1, we reemphasize that the strain gages are no longer positioned at the center of the bars. It is worth comparing the above recommendations with a conventional SPHB system that follows Kolsky's original design. In the case of bars of the same diameters and mechanical properties, the total length of a conventional system is about $l_{s t}+l_{\text {in }}+l_{\text {out }}=5 l_{s t}$. Assuming $D_{\text {in }}<<l_{s t}$, the length of the proposed compact SHPB system is only $l_{s t}+l_{\text {in }}+l_{\text {out }}=3 l_{s t}$. In other words, for the same experimental duration, the compact system is about $40 \%$ shorter than a conventional SHPB system.

\section{Demonstration}

We make use of $40 \mathrm{~mm}$ diameter aluminum input and output bars to demonstrate the validity of the proposed data processing method. Both bars are $2500 \mathrm{~mm}$ long. A first strain gage is positioned on the input bar at a distance of $350 \mathrm{~mm}$ from the input barstriker interface, while a second strain gage is positioned near the center of the input bar. Only one strain gage is glued on the output bar at a distance of $300 \mathrm{~mm}$ from the specimen-output bar interface. Compression experiments are performed on a cylindrical

lead specimen (diameter $25.5 \mathrm{~mm}$, length $6.5 \mathrm{~mm}$ ) at a strain rate of a about $300 / \mathrm{s}$. Two different striker bars will be employed to demonstrate the validity of the modified Kolsky formulas for experiments of short duration (where the reflected wave can be measured directly) and long duration (where a direct measurement of the reflected wave is not possible).

\subsection{Conventional experiment of short duration}

A $800 \mathrm{~mm}$ long aluminum striker bar is used to apply the dynamic loading at a striker speed of $3.9 \mathrm{~m} / \mathrm{s}$ for a duration of about $400 \mu \mathrm{s}$ (including the rise and fall time of the incident wave). Figure 2a shows the incident and reflected strain histories as recorded at 
the center of the input bar along with the transmitted wave recording for the output bar. Since the input bar is more than twice as long as the striker bar (made from the same material), the incident and reflected waves do not superpose at the center of the input bar. Note that the maximum duration for valid measurements of the reflected wave is about $420 \mu \mathrm{s}$ for the $2500 \mathrm{~mm}$ long aluminum input bar. After reconstructing the waves and evaluating the stress histories at the specimen boundaries (taking material and geometric dispersion into account), we calculate the input and output force based on the Eqs. (2) and (4). Their comparison in Fig. $2 \mathrm{~b}$ demonstrates that the assumption of quasi-static equilibrium is approximately satisfied. Subsequently, we compute the stress and strain histories within the specimen using

1. the transported strain histories measured by the first strain gage on the input bar along with the modified Kolsky formulas (9) and (10),

2. the transported strain histories measured by the second (central) strain gage on the input bar along with the three-wave formulas (6) and (7).

The corresponding stress-strain curves are shown in Fig. 3. Both curves are in good agreement which is seen as a partial validation of the proposed processing method.

\subsection{Experiment of long duration}

A $2500 \mathrm{~mm}$ long steel striker bar (diameter $40 \mathrm{~mm}$ ) is used for the second experiment. The striker is launched at a speed of $2.35 \mathrm{~m} / \mathrm{s}$ in order to achieve a similar rate of loading (at the specimen level), with an almost equal amplitude of the incident pulse, as in the previous experiment. However, unlike in the previous experiment, the duration of the loading pulse now exceeds the maximum duration for a valid measurement of the reflected wave, i.e. the incident and reflected wave overlap at the center of the input bar. Standard SHPB processing methods are therefore no longer applicable. Using the strain gage near the striker-input bar interface, the incident wave can be directly measured over a duration of up to about $850 \mu$ s. The stress-strain curve as determined with the modified Kolsky formulas (9a) and (10a) is shown in Fig. 4. 
We make use of the wave deconvolution method proposed by Bussac et al. (2002) to validate the measured stress-strain curve. This method operates in the frequency domain; it is used to reconstruct all waves in the input bar based on the recordings of the first and second input bar strain gage. After calculating the strain histories at the specimen boundaries, the three-wave formulas are used to compute the stress-strain curve. The corresponding result is shown as a red curve in Fig. 4. It follows closely the previous result and is therefore seen as another partial validation of the proposed incident wave based processing method.

\section{Conclusions}

The duration of a split Hopkinson pressure bar experiment is limited by the duration of the loading pulse plateau which is a function of the striker bar length. The length of the input bar needs to be at least as long as the striker bar (if the same material is used) to avoid any shortening of the duration of the loading pulse plateau. In conventional SHPB systems, the input bar is at least twice as long as the striker bar to allow for valid measurements of the stress-strain curve for the specimen material. Song et al. (2007) positioned the input bar strain gage near the impact end to increase-the duration of valid SHPB measurements. To emphasize the advantages of this configuration, and inspired by Kolsky's work (1949), we propose a simple signal processing formula which expresses the stress and strain history in the specimen as a function of the incident and transmitted waves only. It is thus sufficient to measure the incident strain wave in the input bar, while the measurement of the reflected wave may be omitted. The optimal position of the input bar strain gage moves from the center to the striker-input bar interface. At this position, the incident strain wave may be measured for the full duration of the loading even if the input bar is not longer than the striker bar. This observation may be explored either to build compact SHPB systems or to increase the duration of valid measurements for a system of a given length. Two examples are presented to demonstrate the validity of the

proposed modified Kolsky formulas for SHPB experiments. It is emphasized that the proposed configuration is only valid for conventional SHPB experiments where the specimen is in quasi-static equilibrium. 


\section{References}

Bussac MN, Collet P, Gary G, Othman R, An optimization method for separating and rebuilding one-dimensional dispersive waves form multi-point measurements. Application to elastic or viscoelastic bars, Journal of the Mechanics and Physics of Solids, 50:321-349, 2002.

Davies, RM (1948), A critical study of Hopkinson pressure bar. Phil. Trans. Roy. Soc., A240, 375-457.

Duffy J., Campbell J.D., and Hawley, R.H., (1971), On the use of a torsional split Hopkinson bar to study rate effects in 1100-0 aluminium, J. Appl. Mech. 38, 83-91

Gray III GT (2000). Classic Split Hopkinson pressure bar testing; ASM Handbook Vol 8, Mechanical testing and evaluation. ASM 2000:462-476.

Harding J., Wood E.D., and Campbell J.D., (1960), Tensile testing of materials at impact rate of strain, J. Mech. Eng. Sci., 2, 88-96

Hopkinson B. (1913). A method of measuring the pressure in the deformation of high explosives by the impact of bullets. Phil. Trans. Roy. Soc. A213, 437-452

Kolsky H (1949). An investigation of the mechanical properties of materials at very high rates of loading. Proc Phys Soc (London) 63:676-700

Song, B, Chen, WW, Lu, W-Y (2007). Mechanical characterization at intermediate strain rates for rate effects on an epoxy syntactic foam, Int. J. Mech. Sciences 49, 1336-1343. 


\section{Figures}

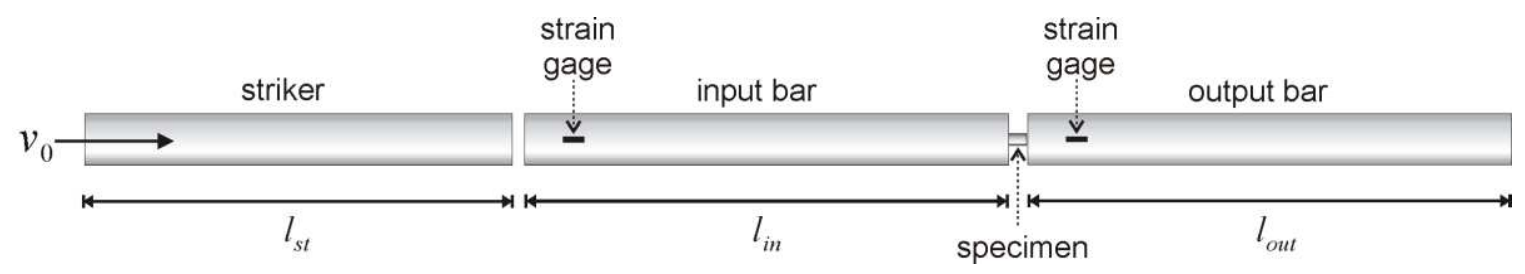

Figure 1. Compact SHPB system composed of three bars of (almost) equal length and two strain gages positioned near the striker-input bar interface and the specimen-output bar interface. 


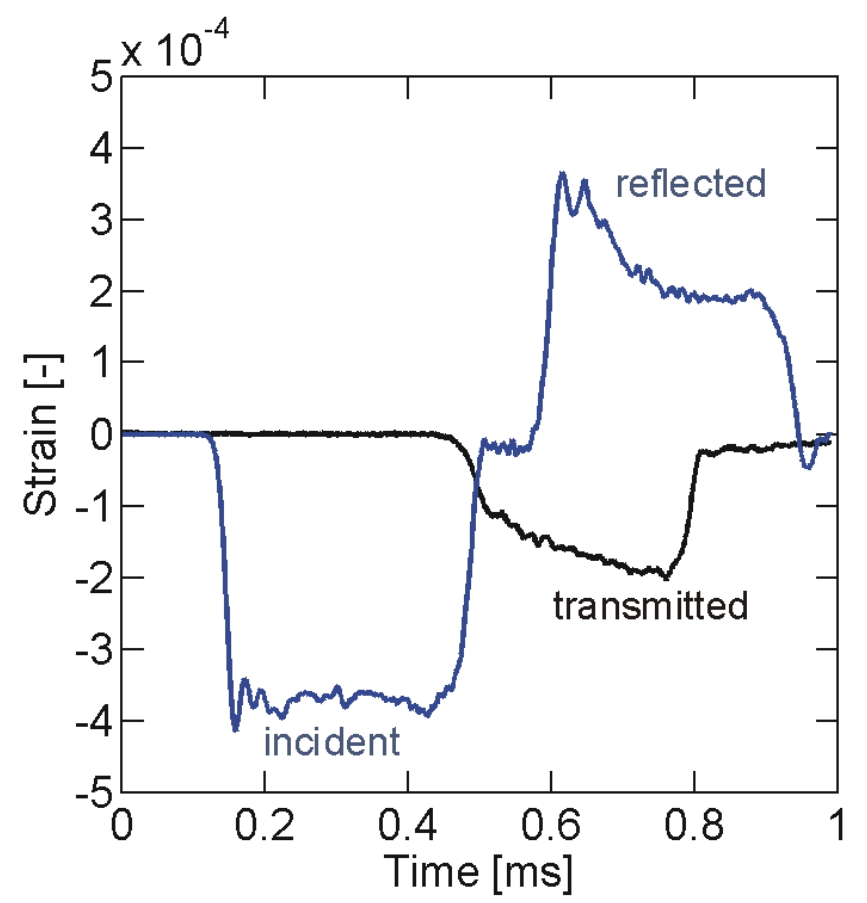

(a)

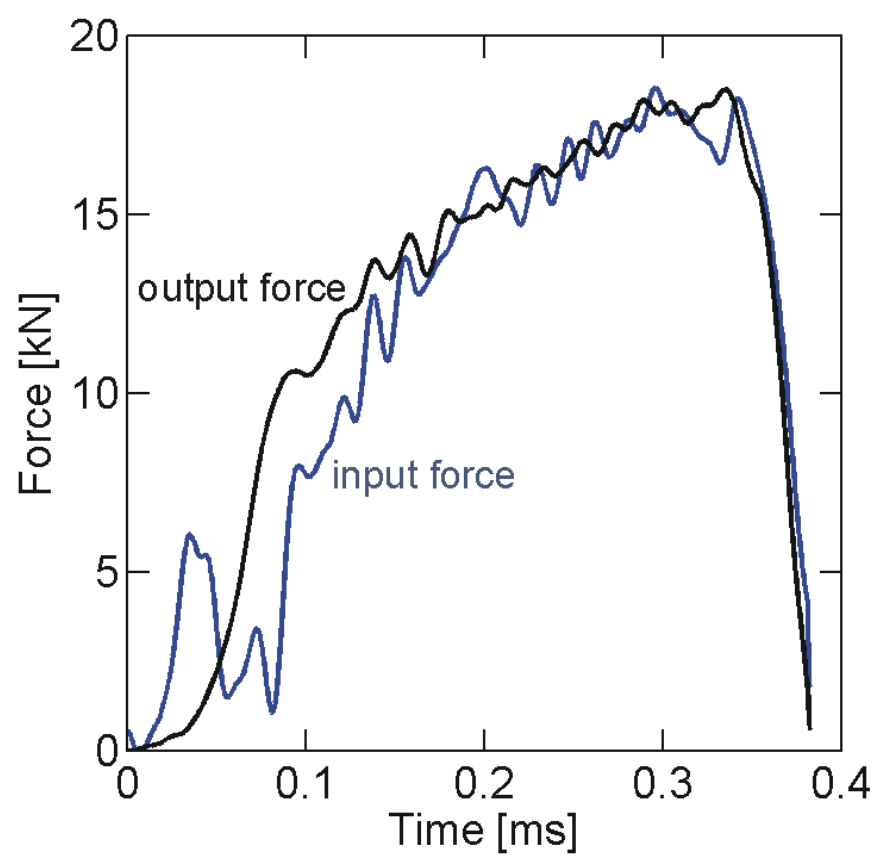

(b)

Figure 2: (a) Recorded strain histories for the incident, reflected and transmitted waves; (b) Corresponding force histories at the specimen boundaries in contact with the input and output bars. 


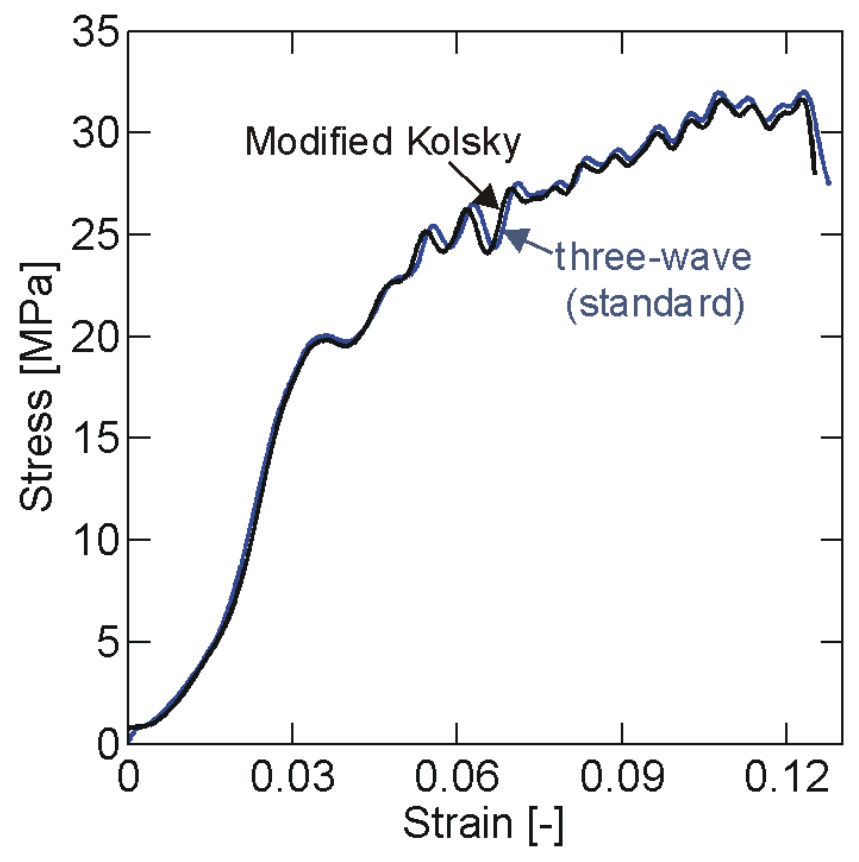

Figure 3: Experiment of short duration: Stress-strain curve as determined using the standard method (based on measurement of incident, reflected and transmitted waves) and the proposed modified Kolsky formulas (based on measurements of the incident and transmitted waves only). 


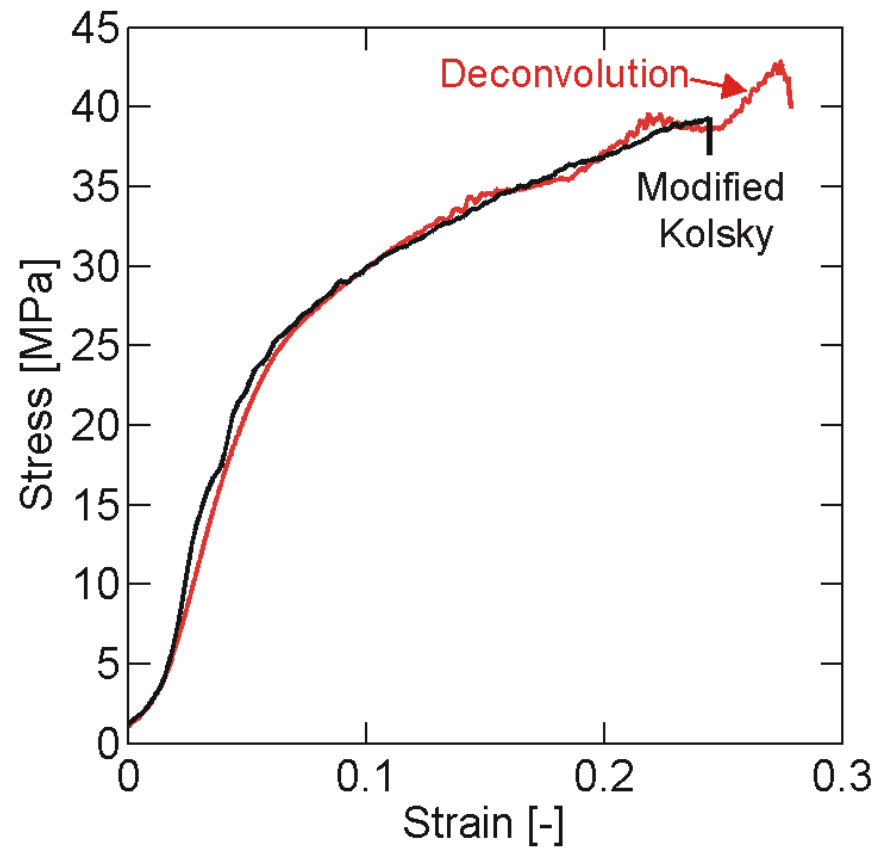

Figure 4: Experiment of long duration: Stress-strain curve as determined using a wave deconvolution method (based on two strain history measurements on the input bar and the transmitted wave) and the proposed modified Kolsky formulas (based on measurements of the incident and transmitted waves only). 